\title{
Default change nudges Covid-19 vaccine uptake: a randomized controlled trial
}

Katya Tentori, $\mathrm{PhD}^{1,2}$; Stefania Pighin, $\mathrm{PhD}^{1}$; Giulia Giovanazzi, ${ }^{3} \mathrm{MD}$; Andrea Grignolio, $\mathrm{PhD}^{4,5}$; Ben Timberlake, $\mathrm{PhD}^{1}$; Antonio Ferro, $\mathrm{MD}^{3}$

${ }^{1}$ Center for Mind/Brain Sciences (CIMeC), University of Trento

${ }^{2}$ Centre for Medical Sciences (CISMed), University of Trento

${ }^{3}$ Provincial Authority for Health Services (APSS), Autonomous Province of Trento (Italy)

${ }^{4}$ International MD Program, Vita-Salute San Raffaele University;

${ }^{5}$ Interdepartmental Center for Research Ethics and Integrity, National Research Council (CNR)

\begin{abstract}
Although vaccination against SARS-CoV-2 is considered the central strategy against the pandemic, uptake lags behind target rates. To explore whether this rate could be enhanced by a nudging strategy that exploits the status quo bias, we conducted a randomized controlled study in northern Italy comparing vaccination acceptance among 2,000 adults aged 50-59 who were either invited to set an appointment (opt-in group) or assigned an individual appointment (opt-out group). Results indicate an increase in vaccination rate for the opt-out group compared to the opt-in group of 3.2 percentage points - a 32\% relative increase. This suggests that a significant portion of those who remain unvaccinated may not hold strong beliefs against vaccination, but rather tend to inaction and may therefore be nudged toward vaccination with a reduction of action required.
\end{abstract}




\section{Introduction}

Although vaccination is acknowledged as the most promising way to reduce the risk of severe Covid-19 infection, ${ }^{1,2}$ areas of higher vaccine hesitancy threaten global efforts to bring the pandemic under control. ${ }^{3,4}$ In this study, we investigated whether Covid-19 vaccine uptake could be increased by exploiting the status quo bias, ${ }^{5}$ that is, the tendency to maintain the current state of affairs, even when it has not been actively chosen and change requires only minimal action. Specifically, we compared the Covid-19 vaccination rate following two default options: one in which vaccination is available with opting-in required to vaccinate (the standard option in many if not all countries), and one in which the vaccination appointment has been scheduled with optingout possible if a person does not want to receive the vaccination.

\section{Method}

Our study involved 2,000 adults aged 50 to 59 (see Figure 1) residing in the Autonomous Province of Trento (Italy). We focused on this population because its constituents were found to be particularly reluctant to vaccinate in the preceding months (see Figure 2), even though they were at relatively high risk of severe disease $\left(0.6 \%\right.$ death rate for Covid- $\left.19^{6}\right)$. Exclusion criteria included professionals subject to mandatory Covid-19 vaccination requirements (e.g., physicians or nurses), residents of extended-care facilities (where vaccination is arranged internally), individuals who had already received or scheduled an appointment for the Covid-19 vaccination and those for whom vaccination was not recommended due to prior Covid-19 infection.

In the Autonomous Province of Trento, Covid-19 vaccination is managed by the APSS (Provincial Authority for Health Services). Participants were assigned to one of two groups obtained by gender- and age-stratified random sampling out of the APSS database. All were sent, by regular mail, a letter from the prevention department of the APSS reminding them of the importance and availability of Covid-19 vaccination. The letter was identical for the two groups, apart from appointment scheduling information. For the opt-in group, this section constituted instructions on how to schedule an appointment for the vaccination (via web or by contacting their family doctor),

while, for the opt-out group, this section comprised date, time and location information for a scheduled vaccination appointment, together with instructions on how to modify it if desired. All participants were offered the BNT162b2 Pfizer/BioNTech vaccine. 
Figure 1. Flow chart of study participants (and their characteristics)

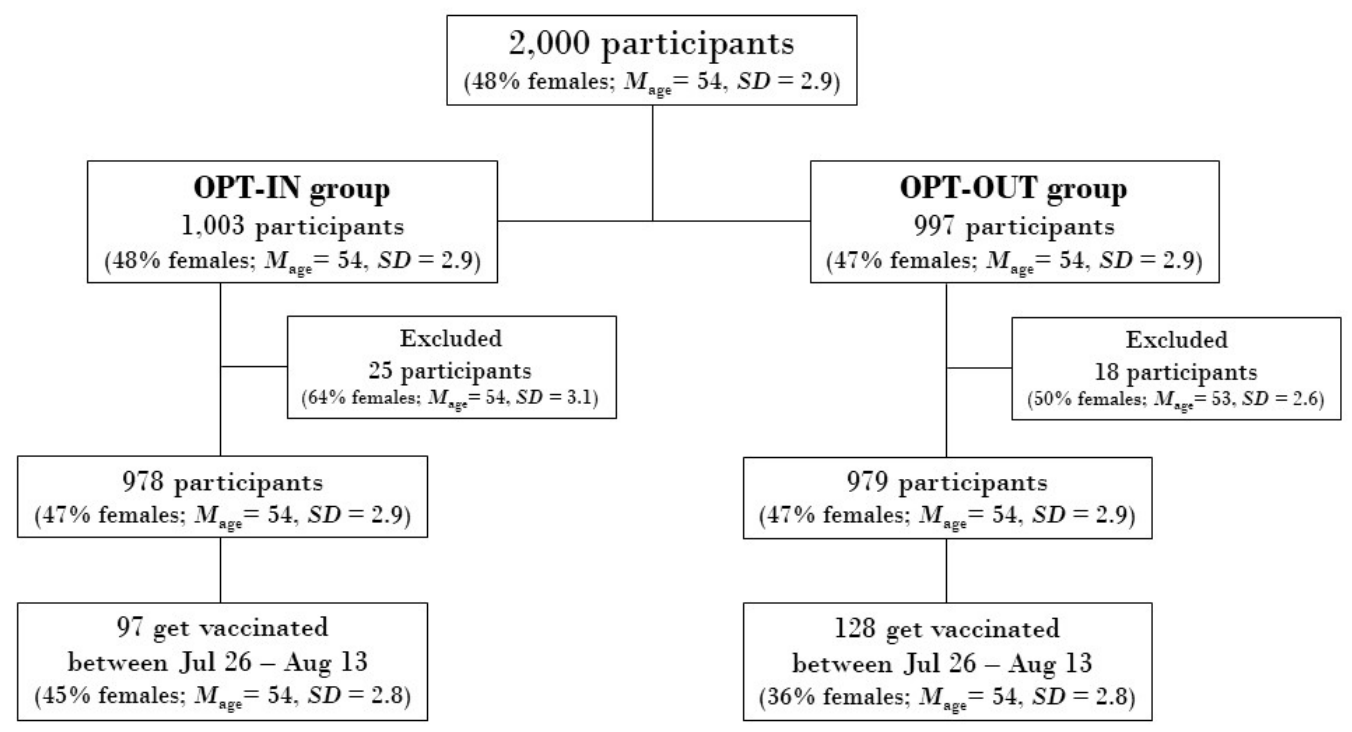

Figure 2. Covid-19 vaccination coverage for individuals aged 50 to 59 in the province of Trento (the target population from which the study sample was drawn) alongside coverage rates for older adults in the same geographical area, as well as the rates for the peer cohort across the entirety of Italy, as of the beginning of data collection (July 26, 2021).

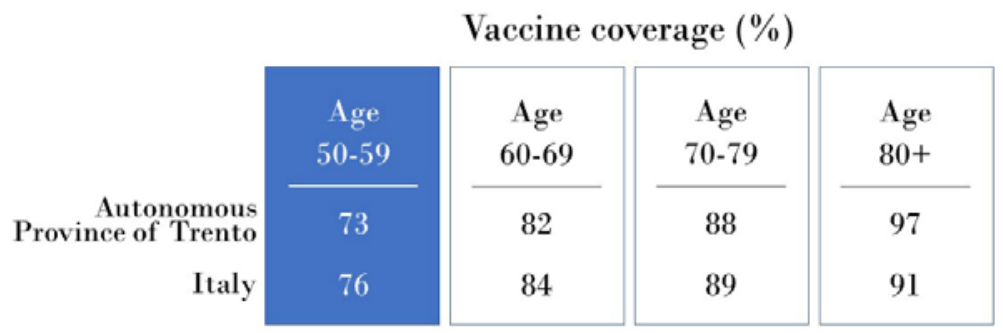

Coders blind to group assignment abstracted vaccination records out of the APSS database for a 19-day interval, between July 26 (i.e., three days after the letters were sent, which is the estimated minimum time for letter delivery and subsequent scheduling of the vaccination) and Aug. 13 (i.e., the last day on which appointments for the opt-out group were scheduled, a Friday before a public holiday weekend). Forty-three participants were excluded because it turned out they had received the Covid-19 vaccination outside the Autonomous Province of Trento shortly before the beginning of the study. The final sample, therefore, comprised 1,957 individuals ( $47 \%$ females; $M_{\text {age }}=54$, $S D=2.9$ ): 978 in the opt-in group and 979 in the opt-out group (see Figure 1). 


\section{Results}

Results showed that 128 of 979 participants in the opt-out group (13.1\%; 95\% confidence interval [CI], 11\%-15.4\%) received the vaccine during the considered interval, compared to 97 of 978 participants in the opt-in group (9.9\%; 95\% CI, 8.1\%-12\%). This difference of 3.2 percentage points is significant $\left(\chi^{2}(1,1,975)=4.79, p=.029\right)$ and represents a $32 \%$ relative increase in vaccination in the opt-out group compared to the opt-in group. Age did not significantly predict the probability of getting vaccinated, while gender did: overall, males were 1.396 (95\% CI, 1.0521.854) times more likely to get vaccinated than females. When controlling for age and gender, the default option was still a significant predictor: participants in the opt-out group were 1.37 (95\% CI, 1.035-1.814) times more likely to get the Covid-19 vaccine than those in the opt-in group.

\section{Discussion}

Prior nudging interventions that promoted vaccination by acting on the default provided encouraging, though variable, results. For example, they have been shown to lead to greater influenza immunization among university workers ${ }^{7}$ and patients ${ }^{8}$ but not among health-care employees. ${ }^{9}$ To our knowledge, this study documents the first application of this specific nudging technique to Covid-19 vaccination efforts, following a number of suggestions for its implementation. ${ }^{10,11}$ Other behavioral nudges — such as incentives, advocacy of personal or social benefits, and reminders designed to make vaccination more salient and easier to book — have been reported to modulate Covid-19 vaccination either not at all or at rates as high as $84 \% .{ }^{12-14}$ Both versions of the letter used in our study could in fact have served as reminders. The obtained results show that, on top of this possible saliency-notice effect, the simple switching of the default from opt-in to opt-out generated significantly greater Covid-19 vaccine uptake. Moreover, it did so in individuals who have previously shown reluctance to vaccinate. These results also indicate that the default can exert a powerful influence on the decision to vaccinate, even when opting-out comes with no cost and does not involve extra effort (since participants could ignore the vaccination appointment with no consequences). Finally, these results confirm that vaccine hesitancy does not necessarily derive from strong concerns about vaccination but may well be rooted in procrastination or in difficulties in following through on the vaccination acquisition procedure. ${ }^{15}$ Notably, this effect of default was obtained despite the measurements being made during the summer holiday season (which, in Italy, peaks between July 15 and Aug. 15) and in the midst of a 
then-new green pass policy (announced in Italy at the end of July 2021 and implemented on Aug. 6). Of course, the acceptability and effectiveness of analogous interventions at other times and in other settings cannot be taken for granted since, as with other nudging strategies, it would require an understanding of all complexities and characteristics of the target populations. ${ }^{16}$ Future research might explore this issue, as well as how to encourage long-term trust and more deliberative adherence to vaccination by combining nudging policies with other strategies, such as education ${ }^{17}$ and communication campaigns ${ }^{18}$ aimed at clarifying the individual and collective benefits of vaccination.

Competing interests: The authors declare no conflict of interest related to this study. A.F. is the director of the APSS, G.G. is employed in the same institution. The research was conducted independently from funders: they had no role in study design, collection, analysis, interpretation of data, writing of the report, or the decision to submit the article for publication.

\section{References}

1. WHO. Covid-19 vaccines. November 2020. (https://www.who.int/emergencies/diseases/novel-coronavirus2019/covid-19-vaccines).

2. Excler JL, Saville M, Berkley S, Kim JH. Vaccine development for emerging infectious diseases. Nat Med 2021;27:591-600.

3. Machingaidze S, Wiysonge CS. Understanding COVID-19 vaccine hesitancy. Nat Med 2021;27:1338-1339.

4. Sallam M. COVID-19 vaccine hesitancy worldwide: a concise systematic review of vaccine acceptance rates. Vaccines 2021;9:160.

5. Samuelson W, Zeckhauser R. Status quo bias in decision making. J Risk Uncertain 1998;1:7-59.

6. Statista. Coronavirus (COVID-19) death rate in Italy as of November 10, 2021, by age group. (https://www.statista.com/statistics/1106372/coronavirus-death-rate-by-age-group-italy/).

7. Chapman GB, Li M, Colby H, Yoon H. Opting In vs Opting Out of Influenza Vaccination. JAMA 2010;304:4344.

8. Milkman KL, Patel MS, Gandhi L, et al. A megastudy of text-based nudges encouraging patients to get vaccinated at an upcoming doctor's appointment. PNAS 2021;118:1-3.

9. Lehmann BA, Chapman GB, Franssen FME, Kok G, Ruiter RAC. Changing the default to promote influenza vaccination among health care workers. Vaccine 2016;34:1389-1392. 
10. Reñosa MDC, Landicho J, Wachinger J, Dalglish SL, Bärnighausen K, Bärnighausen T, McMahon SA. Nudging toward vaccination: a systematic review. BMJ global health 2021;6: e006237.

11. Schwartz J, Holzwarth A. Insights from behavioural economics for effective leadership during the pandemic. BMJ Leader 2021;5:214-218.

12. Berliner Senderey A, Ohana R, Perchik S, Erev I, Balicer R. Encouraging Uptake of the COVID-19 Vaccine Through Behaviorally Informed Interventions: National Real-World Evidence From Israel. 2021;Available at SSRN 3852345.

13. Campos-Mercade P, Meier AN, Schneider FH, Meier S, Pope D, Wengström E. Monetary incentives increase COVID-19 vaccinations. Science 2021;374:879-882.

14. Dai H, Saccardo S, Han MA, et al. Behavioural nudges increase COVID-19 vaccinations. Nature 2021;597:404409.

15. Brewer NT, Chapman GB, Rothman AJ, Leask J, Kempe A. Increasing vaccination: putting psychological science into action. Psychol Sci Public Interest 2017;18:149-207.

16. Saban M, Myers V, Shetrit SB, Wilf-Miron R. Issues surrounding incentives and penalties for COVID-19 vaccination: The Israeli experience. Prev Med 2021;153:106763.

17. Tentori K, Passerini A, Timberlake B, Pighin S. The misunderstanding of vaccine efficacy. Soc Sci Med 2021;289:114273.

18. Chevallier C, Hacquin AS, Mercier H. COVID-19 vaccine hesitancy: Shortening the last mile. Trends Cogn Sci 2021;25:331-333. 